\section{Radioquimioterapia adyuvante en cáncer gástrico completamente resecado: experiencia del Instituto Nacional del Cáncer de Chile}

\author{
NICOLÁS ISA O. ${ }^{1}$, MOISÉS RUSSO N. ${ }^{2,3}$, HERNÁN LÓPEZ V.a
}

\section{Adjuvant chemoradiotherapy in advanced gastric cancer. Experience in 168 patients}

Background: Gastric cancer is one of the most lethal tumors in the Chilean population. Aim: To report the results of adjuvant chemoradiotherapy in advanced gastric cancer. Material and Methods: Review of medical records of patients with locoregionally advanced gastric cancer, subjected to a curative resection and treated with adjuvant chemoradiotherapy. The treatment was based on the INT 0116/SWOG protocol, which includes 5-fluorouracil as a single agent. Patients were followed for a median of 58 months. Results: the records of 168 patients (99 men) treated between 2004 and 2011, were reviewed. Median survival was 41 months. Median lapses between surgery and onset of chemo and radiotherapy were 12 and 17 weeks, respectively. Overall three and five years survival was 53 and $41 \%$, respectively. On multivariate analysis the factors associated with a lower survival were an antral location of the tumor, presence of signet ring cells and more than 15 involved lymph nodes. Conclusions: Three and five years survival of gastric cancer patients subjected to adjuvant chemoradiotherapy was 53 and $41 \%$ respectively. These results are similar to those reported elsewhere.

(Rev Med Chile 2014; 142: 199-203)

Key words: Drug therapy; Radiotherapy; Stomach neoplasms.

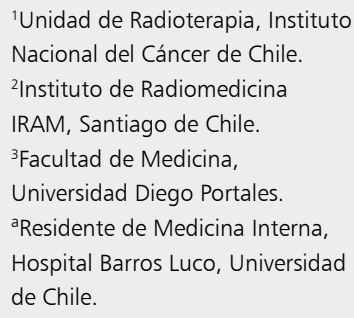

No recibimos apoyo financiero alguno.

Los autores declararon no tener
conflictos de intereses.

Recibido el 3 de agosto de 2013, aceptado el 27 de enero de 2014.

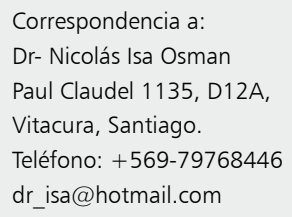

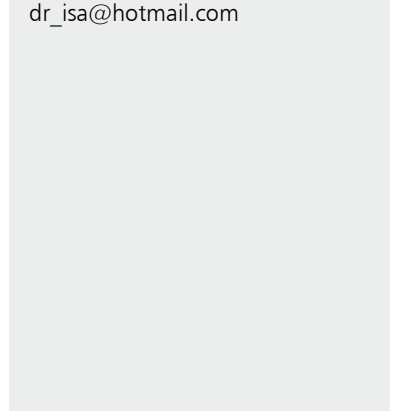

E l cáncer en la región de América Central, Caribe y Sudamérica, es la $2^{\circ}$ causa de muerte, en donde el cáncer gástrico (CG) para los hombres y mujeres es el $3^{\circ}$ más frecuente y el $3^{\circ}$ más mortal. En el caso particular de Chile, el CG para los hombres es el $2^{\circ}$ más frecuente y el $1^{\circ}$ más mortal, para las mujeres es el $4^{\circ}$ más frecuente y el $3^{\circ}$ más mortal ${ }^{1}$, su tasa de mortalidad es de 20 personas cada 100.000 habitantes, la cual se ha mantenido estable desde los años $80^{2}$. El CG tiene una relación hombre/mujer de 2,6:1 y los grupos de edad más afectados son la séptima y octava década, con un promedio de 65 años $^{2}$, es decir afecta a una importante proporción de la población que está aún en etapas activas de la vida. Rara vez es diagnosticado en etapa temprana, ya que sus sín- tomas son vagos e inespecíficos por lo que lleva a un diagnóstico tardío ${ }^{2}$. El tratamiento estándar es la cirugía, pero los resultados obtenidos son modestos, particularmente cuando hay compromiso de Serosa y/o Linfonodos ${ }^{2}$. En estas situaciones el pronóstico de sobrevida a 5 años con compromiso seroso es de $40 \%$ y de $10-47 \%$ cuando involucra linfonodos ${ }^{2}$. Los sitios de falla luego de la cirugía de intención curativa son principalmente intra abdominales: sitio de anastomosis, lecho tumoral $y$ linfonodos regionales en $40-65 \%{ }^{3}$. El esfuerzo de disminuir estas fallas con más cirugía, extendiendo la linfadenectomía, no se ha traducido en mejoría de los resultados en sobrevida libre de enfermedad, sólo ha incrementado la morbi/mortalidad ${ }^{4-7}$. Dada la magnitud del problema, se han investi- 
gado diversas terapias adyuvantes al tratamiento estándar. Si sabemos que, estudios clínicos clásicos han demostrado, con dosis de 45-50 Gy se puede controlar el $90 \%$ de la enfermedad subclínica ${ }^{8,9}$, y esto en teoría aumenta al combinar quimioterapia en dosis radiosensibilizante. No nos es extraño que el uso de Radioquimioterapia adyuvante ha disminuido las fallas locorregionales y mejorado la sobrevida tanto en estudios institucionales chilenas ${ }^{10}$ como internacionales ${ }^{11}$, y a la vez comprobado en randomizados multicéntricos internacionales ${ }^{12,13}$, y comprobado en metanálisis ${ }^{14,15}$. Nos pareció de interés investigar los resultados obtenidos con el uso de estas terapias en nuestro centro, tratados con Radioterapia (RT) y Quimioterapia (QT) adyuvante tal como asi lo indica el estándar mundial.

\section{Material y Métodos}

Se revisaron retrospectivamente las historias clínicas de los pacientes con CG loco-regionalmente avanzados (pT1-4, N0-3, M0), completamente resecados y que fueron derivados desde diversos centros hospitalarios, de Santiago y Regiones, que cumplían con la necesidad de recibir tratamiento (serosa comprometida y/o ganglios comprometidos) adyuvante con RT y QT concomitante en base a 5FU (esquema del INT 0116/SWOG) en el Instituto Nacional del Cáncer de Santiago desde agosto de 2004 a diciembre de 2011. Se encontraron a través del registro electrónico de ficha a 168 pacientes, 99 hombres y 69 mujeres respectivamente. Las características de los pacientes se presentan en la Tabla 1.

La mediana de edad diagnóstica fue de 62 años. El 99\% de los pacientes se encontraban ECOG 0-1. El tratamiento consistió en gastrectomía total, subtotal o esófago-gastrectomía con linfadenectomía D1 (14 pacientes, $8 \%$ ), D2 (153 pacientes, $91 \%$ ) y D3 (1 paciente, $1 \%$ ). La QT consistió en un ciclo de $5 \mathrm{FU}\left(425 \mathrm{mg} / \mathrm{m}^{2} /\right.$ día $)$ y leucovorina $\left(20 \mathrm{mg} / \mathrm{m}^{2} /\right.$ día) por 5 días que comenzó el día 1 y posterior a esto RTQT $5 \mathrm{FU}$ ( $400 \mathrm{mg} / \mathrm{m}^{2} /$ día) y leucovorina $\left(20 \mathrm{mg} / \mathrm{m}^{2} /\right.$ día) por 4 días durante la primera semana y por 3 días la quinta semana de RT, luego dos ciclos mensuales separados por 1 mes de $5 \mathrm{FU}$ ( $425 \mathrm{mg} / \mathrm{m}^{2} /$ día) y leucovorina $\left(20 \mathrm{mg} / \mathrm{m}^{2} /\right.$ día $)$ por 5 días o solamente RTQT $5 \mathrm{FU}$ ( $400 \mathrm{mg} / \mathrm{m}^{2} /$ día $)$ y leucovorina $\left(20 \mathrm{mg} / \mathrm{m}^{2} /\right.$ día $)$ por 4 días durante la primera semana y por 3 días la quinta semana de
RT. Las dosis se ajustaron según toxicidad, medida por CTCAE v4.03, esta se ejecutó de acuerdo al protocolo INT 0116/SWOG, sus características se adaptaron según criterio del oncólogo médico tratante. Las características de la QT programada y finalmente recibida se adjuntan en la Tabla 2.

La RT consistió en RT conformacional 3D con acelerador de alta energía (6-18MV) con 2-7 campos de tratamiento (mediana de 3), dosis de $45 \mathrm{~Gy}$ en 25 fracciones sobre lecho, ganglios regionales y anastomosis. Para los análisis y revisión de ficha de forma retrospectiva, se contó con la autorización del Comité de Ética local del Instituto Nacional del Cáncer.

El análisis de sobrevida se realizó mediante el

Tabla 1. Características de los pacientes

\begin{tabular}{|ll|}
\hline & INC \\
Edad & \\
Mediana & 62 \\
Rango & $(23-77)$ \\
Performance status 0-1 & $99 \%$ \\
Hombres & $59 \%$ \\
T & \\
T1-2 & $15 \%$ \\
T3 & $26 \%$ \\
T4 & $59 \%$ \\
n (+) & \\
0 & \\
1-3 & $20 \%$ \\
$>4$ & $28 \%$ \\
Tipo de gastrectomía & $52 \%$ \\
Subtotal & \\
Total & $27 \%$ \\
Esofago-gastrectomía & $70 \%$ \\
Tipo de disección linfática & $3 \%$ \\
D1 & \\
D2 & $8 \%$ \\
D3 & $91 \%$ \\
\hline
\end{tabular}

Tabla 2. Características de la quimioterapia

\begin{tabular}{|ccc|}
\hline $\begin{array}{c}\text { Ciclos de } \\
\text { quimioterapia }\end{array}$ & $\begin{array}{c}\text { Ciclos } \\
\text { programados }\end{array}$ & $\begin{array}{c}\text { Ciclos } \\
\text { recibidos }\end{array}$ \\
\hline 1 & $0 \%$ & $1 \%$ \\
2 & $19 \%$ & $23 \%$ \\
3 & $3 \%$ & $19 \%$ \\
4 & $0 \%$ & $11 \%$ \\
5 & $78 \%$ & $46 \%$ \\
\hline
\end{tabular}


Radioquimioterapia adyuvante en cáncer gástrico - N. Isa et al

Tabla 3. Análisis estadístico

\begin{tabular}{|lcccc|}
\hline & P univariado & HR & p multivariado & HR \\
Sexo & 0,099 & & & \\
Tabaco & 0,34 & & & \\
Antro más cuerpo & 0,007 & 2,2 & 0,002 & 2,29 \\
Anillo de sello & 0,0061 & 1,75 & 0,044 & 1,53 \\
ILV + & 0,014 & 1,91 & 0,057 & 1,68 \\
N + & 0,0008 & 1,32 & & \\
Cada N + & 0,001 & 1,05 & & 2,11 \\
> 15 Ganglios + & 0,001 & 2,54 & 0,015 & 1,52 \\
\hline Estadio patológico AJCC7 & 0,017 & 1,62 & 0,075 & \\
\hline
\end{tabular}

método de Kaplan-Meier, los datos de sobrevida se obtuvieron a partir de los publicados en el Registro Civil de Chile. Comparaciones de sobrevida entre grupos se realizaron mediante el método de log-rank. Comparaciones de características entre grupos se realizaron mediante t-Student o RankSum según fuese apropiado. Exploraciones de factores pronósticos relevantes se realizaron con análisis univariado y multivariado con regresión de Cox. Grupos de riesgo ganglionares se identificaron mediante una partición recursiva de los residuos de Martingale posterior al modelo de riesgos proporcionales de Cox, en donde se buscó un punto de corte significativo que influenciara la sobrevida. Todos los análisis fueron realizados en STATA 12. Los análisis explorados se resumen en la Tabla 3.

\section{Resultados}

Con una mediana de seguimiento de 58 meses (mínimo de 12 meses), la mediana de sobrevida fue de 41 meses. La mediana desde la cirugía al inicio de la QT fue de 12 semanas y a inicio de la RT fue de 17 semanas. La mayor toxicidad durante la RT, medida por el médico oncólogo radioterapeuta tratante, mediante los criterios RTOG (Radiation Therapy Oncology Group), fue grado 3 en 3,5\% de los pacientes, principalmente gastro intestinal. La sobrevida global a 1, 2, 3, 4, 5 años fue de $88 \%$, $64 \%, 53 \%, 47 \%$ y $41 \%$ respectivamente (Figura $1)$. De los factores explorados que fueron sexo, tabaco, ubicación tumoral, grado histológico, células en anillo de sello, ILV, tipo de cirugía, tipo de disección linfática, T, N, estadio AJCC 7, ganglios examinados, ganglios comprometidos, número de ciclos de quimioterapia, semanas desde la cirugía a quimioterapia, semanas desde la cirugía a radioterapia, aquellos estadísticamente significativos, se resumen en la Tabla 3. Al análisis univariado los asociados a mal pronóstico de sobrevida global fueron: ubicación tumoral en antro más cuerpo ( $\mathrm{p} 0,007)$, presencia de células en anillo de sello (p 0,0061), ILV+ (p 0,014), linfonodos positivos (p 0,0008 ) con un HR de 1,32 por cada estadio de $\mathrm{N}$ que aumenta y un HR de 1,05 por cada ganglio positivo que se encuentre (p 0,001), más de 15 ganglios positivos ( $\mathrm{p} 0,001)$ y mayor grupo de estadio patológico AJCC7 (p 0,017). En el análisis multivariado, los factores estadísticamente significativos asociados a mal pronóstico de sobrevida global fueron: ubicación tumoral en antro más cuerpo ( $p$ 0,002), presencia de células en anillo de sello ( $\mathrm{p}$ 0,044) y más de 15 ganglios positivos

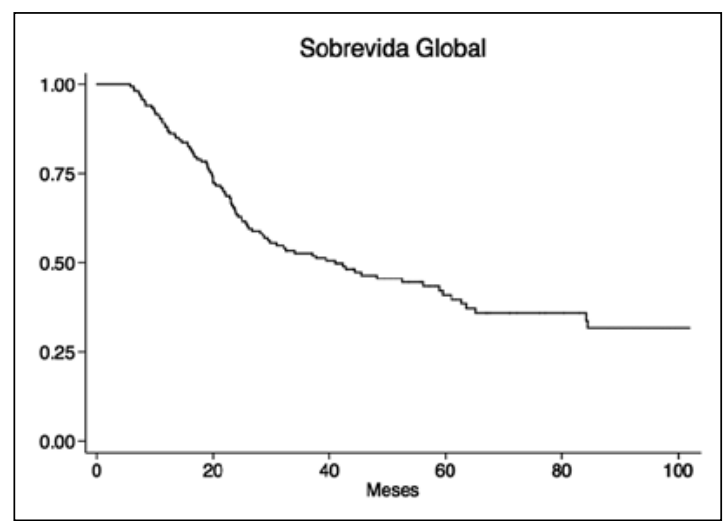

Figura 1. Sobrevida global. 


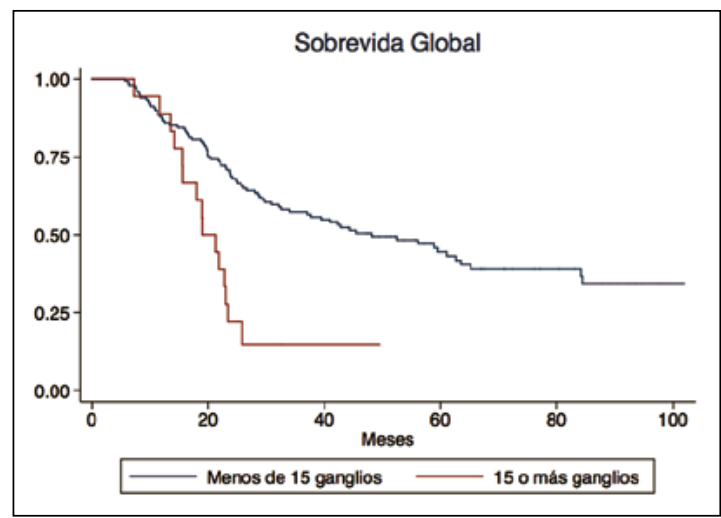

Figura 2. Análisis de partición recursiva según número de ganglios comprometidos. Log rank $p=0,015 ;$ HR 2,54.

(p 0,015). En el análisis de partición recursiva tener menos de 15 ganglios positivos tiene una mediana de sobrevida de 48 meses versus 19 meses al tener más de 15 ganglios positivos (Figura 2).

\section{Discusión}

El tratamiento adyuvante con RTQT en nuestra institución ha mostrado ser efectivo y seguro, dada la baja toxicidad reportada, la cual no influyó en los resultados finales, pese a las 12 semanas de tardanza de inicio de la QT luego de la cirugía o de las 17 semanas de tardanza de inicio de la RT luego de la cirugía, con resultados en sobrevida a 3 y 5 años de $53 \%$ y $41 \%$ respectivamente, resultados comparables con los del estudio del Intergroup $0116^{12}$ y su última actualización del $2012^{13}$, con sobrevidas a 3 y 5 años de $50 \%$ y $41 \%$ respectivamente, esto quizás debido a la mejor calidad de cirugía ofrecida, ya que $91 \%$ de los pacientes recibieron linfadenectomía D2 versus el estudio Intergroup 0116 en que solamente un $10 \%$ de los pacientes recibieron disección D2. En otras palabras, pareciera que es lo mismo realizar una buena cirugía y tratar tardíamente, como en nuestra institución, o realizar una cirugía insuficiente, por no realizar una disección linfática D2, pero recibir una adyuvancia en un tiempo correcto, como en la publicación del Intergroup 0116 y su última actualización $\mathrm{n}^{12,13}$. Así deberíamos perseguir el ideal en nuestro medio, que sería realizar una gastrectomía con disección linfática D2 y recibir el tratamiento en un tiempo prudente y así tratar de emular los resultados del estudio observacional de $\mathrm{Kim}^{11}$ que alcanza una mediana de sobrevida de $57 \%$ a 5 años, muy superior a los resultados nuestros y del INT 0116. Esto teóricamente deberíamos evidenciarlo si es que mejoran nuestros tiempos desde la cirugía al tratamiento adyuvante. Además es interesante y sugerente el impacto de tener un tumor que comprometa antro y cuerpo, presencia de células en anillo de sello, 15 o más linfonodos positivos, resultados que no han sido expresados en otras series.

\section{Referencias}

1. World Cancer Report 2008. International Agency for Research on Cancer. Lyon 2008.

2. Guía clínica Cáncer gástrico MINSAL, Chile 2009 (http://www.minsal.cl/portal/url/item/722233c6b943c d08e 04001011f011d5e.pdf).

3. Gastric cancer NCCN v2.2013 (www.nccn.org).

4. Cuschieri A, Fayers P, Fielding J, Craven J, Bancewicz J, Joypaul V, et al. Postoperative morbidity and mortality after D1 and D2 resections for gastric cancer: preliminary results of the MRC randomized controlled surgical trial. Lancet 1996; 347: 995-9.

5. Bonenkamp JJ, Hermans J, Sasako M, Van de Velde CJ, Welvaart K, Songun I, et al. Extended lymph-node dissection for gastric cancer. N Engl J Med 1999; 340: 908-14.

6. Hartgrink HH, Van de Velde CJH, Putter H, Bonenkamp JJ, Klein Kranenbarg E, Songun I, et al. Extended Lymph Node Dissection for Gastric Cancer: Who May Benefit? Final Results of the Randomized Dutch Gastric Cancer Group Trial. J Clin Oncol 2004; 22: 2069-77.

7. Songun I, Putter H, Kranenbarg EM, Sasako M, van de Velde CJ. Surgical treatment of gastric cancer: 15-year follow-up results of the randomised nationwide Dutch D1 D2 trial. Lancet Oncol 2010; 11: 439-49.

8. Fletcher GH. Clinical dose-response curve of subclinical aggregates of epithelial cells and its practical application in the management of human cancers. In: Friedman M, editor. The biological and clinical basis of radiosensitivity, Springfield: CC Thomas; 1974. p. 485-501.

9. Withers HR, Peters LJ, Taylor JMG. Dose-response relationship for radiation therapy of subclinical disease. Int J Radiat Oncol Biol Phys 1995; 31: 353-9.

10. Baeza M, Giannini O, Rivera R, González P, González J, Vergara E, et al. Adjuvant Radiochemotherapy In The Treatment Of Completely Resected, Locally Advanced 
Gastric Cancer. Int. J. Radiation Oncology Biol Phys 2001; 50: 645-50.

11. Kim S, Lim DH, Lee J, Kang WK, MacDonald JS, Park $\mathrm{CH}$, et al. An observational study suggesting clinical benefit for adjuvant post operatory chemoradiation in a population of over 500 cases after gastric resection with D2 nodal dissection for adenocarcinoma of the stomach. Int J Rad Onc Biol Phys 2005; 63: 1279-85.

12. Macdonald JS, Smalley SR, Benedetti J, Hundahl SA, Estes NC, Stemmermann GN, et al. Chemoradiotherapy after surgery compared with surgery alone for adenocarcinoma of the stomach or gastroesophageal junction. $\mathrm{N}$ Engl J Med 2001; 345: 725-30.

13. Smalley S, Benedetti J, Haller D, Hundahl SA, Estes NC,
Ajani JA, et al. Updated Analysis of SWOG-Directed Intergroup Study 0116:A Phase III Trial of Adjuvant Radiochemotherapy Versus Observation After Curative Gastric Cancer Resection. J Clin Oncol 2012; 30: 2327 33.

14. Valentini V, Cellini F, Minsky B, Mattiucci GC, Balducci M, D’Agostino G, et al. Survival after radiotherapy in gastric cancer: Systematic review and meta-analysis of published literature. Radiotherapy and Oncology 2009; 92: 176-83.

15. Dikken J, Jansen E, Cats A, Bakker B, Hartgrink HH, Kranenbarg EM, et al. Impact of the Extent of Surgery and Postoperative Chemoradiotherapy on Recurrence Patterns in Gastric Cancer- J Clin Oncol 2010; 28: 2430-6. 